\title{
Cardiac biomarkers: a contemporary status report
}

\author{
Alan S Maisel, Vikas Bhalla and Eugene Braunwald
}

Nature Clinical Practice Cardiovascular Medicine (2006) 3: 24-34 [doi:10.1038/ncpcardio0405]

doi:10.1038/ncpcardio0514

\section{CORRIGENDUM}

In the January 2006 issue, the footnote to Table 1 was published as "aN-terminal probrain natriuretic peptide has yet to be approved by the FDA for risk-stratification purposes in acute coronary syndromes". This should have appeared as "aN-terminal pro-brain natriuretic peptide has been approved by the FDA for risk-stratification purposes in acute coronary syndromes and recently also for riskstratification purposes in patients with stable coronary artery disease".

Table 1 The usefulness of inflammatory biomarkers in diagnosis and risk stratification in acute coronary syndromes.

\begin{tabular}{llllllll}
\hline & \multicolumn{7}{c}{ Biomarker } \\
\cline { 2 - 7 } & CK-MB & cTnI/T & hsCRP & $\begin{array}{l}\text { BNPI } \\
\text { NT-proBNP }\end{array}$ & IMA & MPO & CD40L \\
\hline Diagnosis & Yes & Yes & No & No & Yes & $\begin{array}{l}\text { Result } \\
\text { unclear }\end{array}$ & No \\
Outcomes & Death & Death/ & Death/MI/ & Death/CHF & No & Death/MI & Death/MI \\
Short term & Yes & Yes & Yes & Yes & - & Yes & Yes \\
Long term & Yes & Yes & Yes & Yes & - & Yes & Yes \\
Independent & Yes & Yes & Yes & Yes & - & Yes & Yes \\
FDA approval & Yes & Yes & Yes & Yes ${ }^{\text {a }}$ & Yes & No & No \\
Guidelines & Class I & Class I & Class lla & Yes & No & No & No \\
\hline
\end{tabular}

aN-terminal pro-brain natriuretic peptide has been approved by the FDA for risk-stratification purposes in acute coronary syndromes and recently also for risk-stratification purposes in patients with stable coronary artery disease.

BNP, brain natriuretic peptide; CD40L, CD40 ligand; CHF, congestive heart failure; CK-MB, creatine kinase MB; cTnl/T, cardiac troponin I or T; hsCRP, high-sensitivity C-reactive protein; IMA, ischemia-modified albumin; MI, myocardial infarction; MPO,

myelopyroxidase; NT-proBNP, N-terminal pro-brain natriuretic peptide; RI, recurrent infarction. 